\title{
Efficient Intensional Implementation for Lazy Functional Languages ${ }^{\star}$
}

\author{
A. Charalambidis ${ }^{1}$, A. Grivas ${ }^{2}$, N. Papaspyrou ${ }^{2}$, and P. Rondogiannis ${ }^{1}$ \\ 1 Department of Informatics and Telecommunications \\ University of Athens, Panepistimiopolis, 15784 Athens, Greece \\ E-mail: $\{$ a.charalambidis, prondo\}@di.uoa.gr \\ 2 School of Electrical and Computer Engineering \\ National Technical University of Athens, Polytechnioupoli, 15780 Zografou, Greece \\ E-mail: \{agrivas,nickie\}@softlab.ntua.gr
}

\begin{abstract}
The intensional transformation is a technique that can be used in order to eliminate higher-order functions from a functional program by introducing appropriate context manipulation operators. The transformation can be applied to a significant class of higher-order programs and results in equivalent zero-order intensional programs that can be executed in a simple demand-driven way. Despite its simplicity, the transformation has never been seriously evaluated with respect to its efficiency and potential. Certain simple implementations of the technique have been performed, but the questions regarding the merits of the method have remained inconclusive. In this paper we demonstrate that the transformation can be efficiently implemented by using what we call lazy activation records, namely activation records in which some entries are filled on-demand. An evaluation of our implementation demonstrates that the technique outperforms some of the most well-known functional programming systems, for the class of programs that can be transformed.
\end{abstract}

\section{Introduction}

The intensional transformation [Yag84,Ron94,RW97,RW99] is a technique that systematically reduces the order of a source functional program by introducing appropriate context manipulation operators. More specifically, given a functional program of order $M$, the technique in its first step reduces the program to a $(M-1)$-order intensional program; at the second step, the new program is reduced so as to become $(M-2)$-order, and so on. The final outcome is a program that contains only zero-order definitions but which also contains context switching operators that work on $M$ independent dimensions (all these will be further explained and illustrated in the coming sections). The resulting program can be evaluated in a simple demand-driven way, and this gives an interesting scheme for implementing lazy functional languages.

\footnotetext{
* This work has been partially supported by the University of Athens under the project "Kapodistrias" (grant no. 70/4/5827).
} 
The transformation in its present form can be applied to functional languages that allow function names to be passed as parameters in function calls. At present it remains an open problem whether the technique can be generalized so as to be applicable to a more general class of higher-order functional programs, e.g. including partial application of functions (see the concluding section of [RW99] for a further discussion on this issue).

Despite the above restriction, the technique can be used to implement some useful and non-trivial functional programming languages. Experimental implementations of the transformation have been undertaken in the past. In [RW93] an experimental interpreter and a compiler are described, based on the intensional approach and appearing to be relatively efficient. However, both systems are based on a technique that extensively uses hashing at run-time, and this makes the performance of the system to be unpredictable in some cases. Moreover, this hashing-based approach requires a garbage collection process which is not always very accurate, and also imposes a significant time-overhead to the execution. Conclusively, the whole idea appeared to be simple and easy to implement, but not viable as a serious implementation framework. In [RW94b] it is proposed that the runtime of the intensional technique can be adapted to work along the lines of traditional activation records. Of course, there is a significant difference, namely that each activation record should now contain information that would enable the context manipulation operators of the target program to work properly. This approach appeared to be much more promising than the hashing-based one. First-order programs appeared to run as efficiently as the best functional implementations available at that time; however, as the order of the programs increased, the performance of the technique seemed to degrade.

In this paper we refine and extend the technique of [RW94b]. More specifically, we extend the activation records of [RW94b] to contain additional information related to the higher-order functions of the initial program. The resulting implementation behaves in a much more uniform way than all the previous attempts. Moreover, our current system outperforms most of the existing compilers for lazy functional languages, for the class of programs that can be transformed.

\section{The Intensional Transformation Technique}

The intensional transformation technique can be applied to higher-order functional programs with the following two restrictions:

1. There are no functions returning functions, i.e. the body of every function definition is zero-order.

2. Partial application of functions is not allowed, i.e. a function name can only be called with the right number of actual parameters (resulting in a zeroorder value) or passed as a parameter to another function.

For example, the following (in Haskell syntax) is a legitimate program:

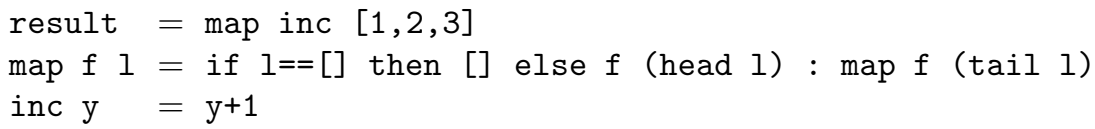


but the following is not an acceptable program, because of the partial application add $(s \mathrm{x})$ in the definition of $\mathrm{f}$ :

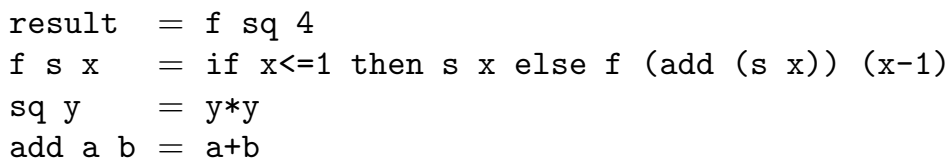

Of course, there exist many programs of the second form that can be preprocessed so as to become programs of the first form, but this will not concern us any further here. In the rest of this paper, we only consider higher-order functional programs that obey the two aforementioned restrictions and, as partial application is not allowed, we use a Pascal-like notation with parentheses in function calls for clarity (e.g. $f(s, x)$ instead of $f s x)$. Moreover, for simplicity reasons, we assume that function parameters have unique names.

In the rest of this section we give an intuitive introduction to the intensional transformation technique. The main idea of the transformation is that an $M$-order functional program can first be transformed into an $(M-1)$-order intensional program, i.e. a program that uses functions of order at most $(M-1)$ and which is enriched with context manipulation operators. The same procedure can then be repeated for the new program, until we finally get a zero-order intensional program. As the transformation consists of a number of steps, we use a different set of operators for each step. For the first step we use the operators case $^{M-1}$, actuals ${ }_{i}^{M-1}$ and $\operatorname{call}_{i}^{M-1}$, where $i$ ranges over the natural numbers. For the second step we use case ${ }^{M-2}$, actuals ${ }_{i}^{M-2}$ and $\operatorname{call}_{i}^{M-2}$, and so on. The semantics of these operators will be described shortly.

The final program that results from the transformation is zero-order, i.e. it consists solely of nullary variable definitions. The value of this program can be computed by evaluating its top-level variable result with respect to a context (or $t a g$ ). For an initial program of order $M$, a tag is an $M$-sequence (a sequence of length $M$ ) of lists, where each list corresponds to a different order of the program. The operators we mentioned above operate on these $M$-dimensional tags. Before we proceed with the details of the execution, we give an example of the transformation.

\subsection{The transformation algorithm}

Consider the following simple second-order program:

$$
\begin{array}{ll}
\text { result } & =\mathrm{f}(\text { inc }, 2) \\
\mathrm{f}(\mathrm{g}, \mathrm{y}) & =\mathrm{g}(\mathrm{y}) * \operatorname{apply}(\mathrm{g}, \mathrm{y}) \\
\operatorname{apply}(\mathrm{h}, \mathrm{x}) & =\mathrm{h}(\mathrm{x}) \\
\operatorname{inc}(\mathrm{a}) & =\mathrm{a}+1
\end{array}
$$

By an inspection of the program, it is easy to see that the functions $f$ and apply are second-order. The intensional transformation in its first step eliminates all first-order parameters: the first argument of $f$ and the first argument of 
apply disappear from the calling sites and reappear as additional definitions. Furthermore, as witnesses of the disappearing parameters, operators of the form $\operatorname{call}_{i}^{1}$ appear in the calling sites, where the superscript 1 is the order of the parameters that disappeared at this step and the subscript $i$ is simply a counter, one for each function of the source program.

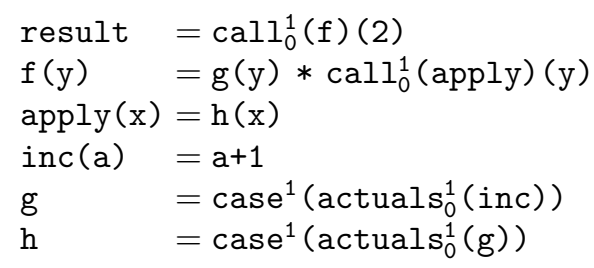

In the above program the definitions of $g$ and $h$ contain the actual parameters that were removed from the calling sites, each prefixed by an intensional operator of the form actuals $s_{i}^{1}$. Both definitions are equations between function expressions. We can change this by introducing new parameters, $\mathbf{z}$ and $\mathbf{w}$ :

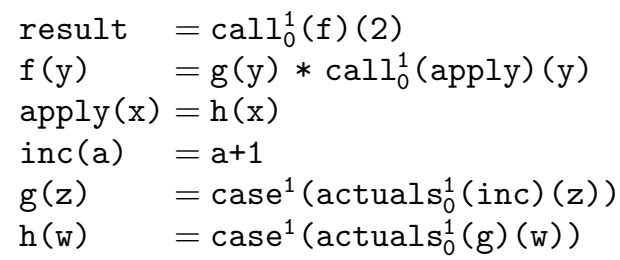

After this step of the transformation, all functions are now first-order (they have only zero-order parameters). A non-standard aspect of this new program is the existence of certain function calls of the form $\mathbf{q}(\mathbf{f})\left(\mathbf{E}_{0}, \ldots \mathbf{E}_{n-1}\right)$ where $\mathbf{q}$ is an intensional operator, e.g. $\operatorname{call}_{0}^{1}(\mathrm{f})(2)$, or actuals $\mathrm{s}_{0}^{1}$ (inc)(z). Such calls will receive a special treatment in the next step of the transformation.

We can now perform the second and final step of the transformation that will remove all zero-order parameters and result in a zero-order intensional program. We proceed as before, the main difference being that we use a new dimension and corresponding new operators. Notice also below the use of the "." syntactic composition operator, which is introduced for notational convenience. In particular, an expression of the form $\mathbf{q}_{1} \cdot \mathbf{q}_{2}$ (f) will be considered equivalent to $\mathbf{q}_{1}\left(\mathbf{q}_{2}(\mathbf{f})\right.$ ), i.e. the composition operator binds stronger than function application.

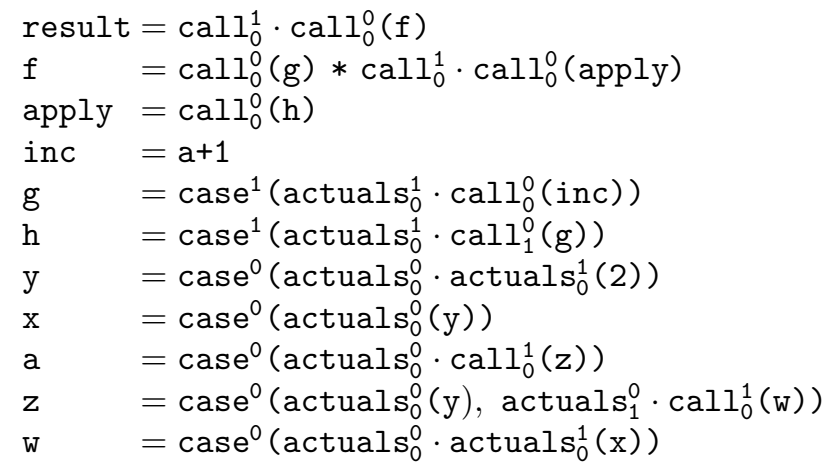


The transformation is similar to the one that took place in the first step, the main difference being the treatment of calls of the form $\mathbf{q}(\mathbf{f})\left(\mathbf{E}_{0}, \ldots \mathbf{E}_{n-1}\right)$. Consider as an example the (generalized) function call $\mathrm{call}_{0}^{1}(\mathrm{f})(2)$ and notice the new expression actuals $\mathbf{s}_{0}^{0} \cdot \operatorname{actual} \mathbf{s}_{0}^{1}(2)$ that appears in the final program corresponding to the actual parameter 2. The new aspect here is the appearance of the operator actuals $s_{0}^{1}$, which we will call the inverse of the operator $\mathrm{call}_{0}^{1}$ that existed in the initial call. In general, the inverse of $\operatorname{call}_{i}^{m}$ is actuals $\mathbf{s}_{i}^{m}$ and vice-versa.

A thorough description of the transformation algorithm is given in [RW99]. Informally, it consists of repeating the following steps until the program becomes zero-order. For each function $\mathbf{f}$ of the current highest order $m$ :

1. Number the textual occurrences of calls to $\mathbf{f}$ in the program, starting at 0 .

2. Remove from the $i$-th call to $\mathbf{f}$ all the actual parameters of order $m-1$. Prefix the call to $\mathbf{f}$ with $\mathrm{call}_{i}^{m-1}$.

3. Remove from the definition of $\mathbf{f}$ the formal parameters of order $m-1$.

4. For every formal parameter $\mathbf{x}$ of $\mathbf{f}$ that was eliminated, introduce a case $e^{m-1}$ definition with as many alternatives as are the calls to $\mathbf{f}$ in the program. More specifically, the $i$-th argument of case ${ }^{m-1}$ corresponds to the $i$-th call to $\mathbf{f}$ in the program, and is an expression starting with actual $\mathbf{s}_{i}^{m-1}$. Moreover, if the particular call to $\mathbf{f}$ is of the form $\mathbf{Q}(\mathbf{f})\left(\mathbf{E}_{0}, \ldots \mathbf{E}_{n-1}\right)$, where $\mathbf{Q}$ is the syntactic composition of a number of intensional operators, the inverse of $\mathbf{Q}$ must be taken into consideration when creating the subexpressions of case $^{m-1}$.

\subsection{Evaluating the transformed program}

Given a source functional program of order $M$, the execution model for the final zero-order program that results from the transformation requires tags to be $M$-sequences of lists of natural numbers, where each list corresponds to one step in the transformation. We will use the notation $\left\langle w_{0}, \ldots, w_{M-1}\right\rangle$ to denote a tag. The evaluation of a program starts with the empty tag: an $M$-sequence of empty lists. The operators $\mathrm{call}_{i}^{m}$ and $\operatorname{actuals}_{i}^{m}$ can now be thought of as operations on tags. The semantics of $\operatorname{call}_{i}^{m}$ can be described as follows: given a tag, $m$ is used in order to select the corresponding list from the tag. The list is then prefixed with $i$ and returned to the tag. On the other hand, actuals ${ }_{i}^{m}$ takes from the tag the list corresponding to $m$, verifies that the head of the list is equal to $i$ and returns the tail of the list to the tag. The case ${ }^{m}$ construct that appears on newly introduced definitions selects the alternative to be evaluated by inspecting the head of the list that corresponds to $m$. More formally, the semantic equations for the intensional operators that appear in the transformed (zero-order) programs are:

$$
\begin{array}{ll}
\operatorname{call}_{i}^{m}(a)\left\langle w_{0}, \ldots w_{m}, \ldots w_{M-1}\right\rangle & =a\left\langle w_{0}, \ldots i: w_{m}, \ldots w_{M-1}\right\rangle \\
\operatorname{actuals}_{i}^{m}(a)\left\langle w_{0}, \ldots i: w_{m}, \ldots w_{M-1}\right\rangle & =a\left\langle w_{0}, \ldots w_{m}, \ldots w_{M-1}\right\rangle \\
\operatorname{case}^{m}\left(a_{0}, \ldots a_{n-1}\right)\left\langle w_{0}, \ldots i: w_{m}, \ldots w_{M-1}\right\rangle & =a_{i}\left\langle w_{0}, \ldots i: w_{m}, \ldots w_{M-1}\right\rangle
\end{array}
$$


Notice that in the case of the actuals $\mathbf{s}_{i}^{m}$ operator, the semantic equation does not specify what happens if the check made by the operator fails. The result in this case is undefined. However, the test performed by actuals $\mathbf{s}_{i}^{m}$ never fails in the case of programs generated by the transformation.

The final zero-order programs that result from the transformation can be executed using an EVAL function, which takes an expression and a tag and returns the result of evaluating this expression under this tag. One can think of $E V A L$ as a simple interpreter that works by following the semantic equations of the intensional operators given above. Moreover, EVAL also performs a simple form of substitution: every time it needs to evaluate a nullary variable of the program under a specific context, it simply replaces the variable with its defining expression and continues the evaluation. Execution of a program starts by demanding the value of result under the empty tag. A complete denotational semantics for the zero-order intensional language that can serve as a basis for $E V A L$ is given in [RW99]. We now demonstrate the execution of the previous program:

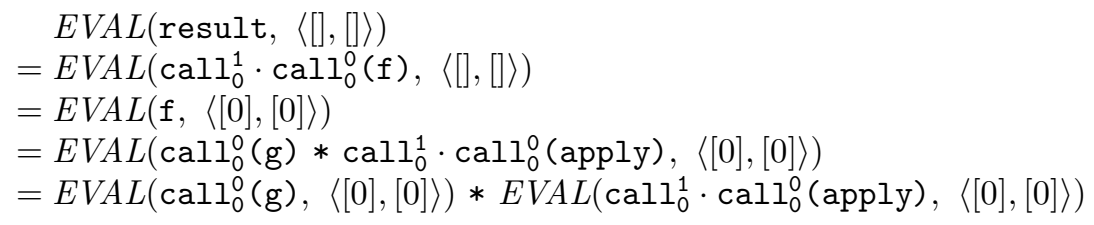

Now, the overall result can be computed by evaluating independently the two expressions and then multiplying the two results. The evaluation of the first expression proceeds as follows:

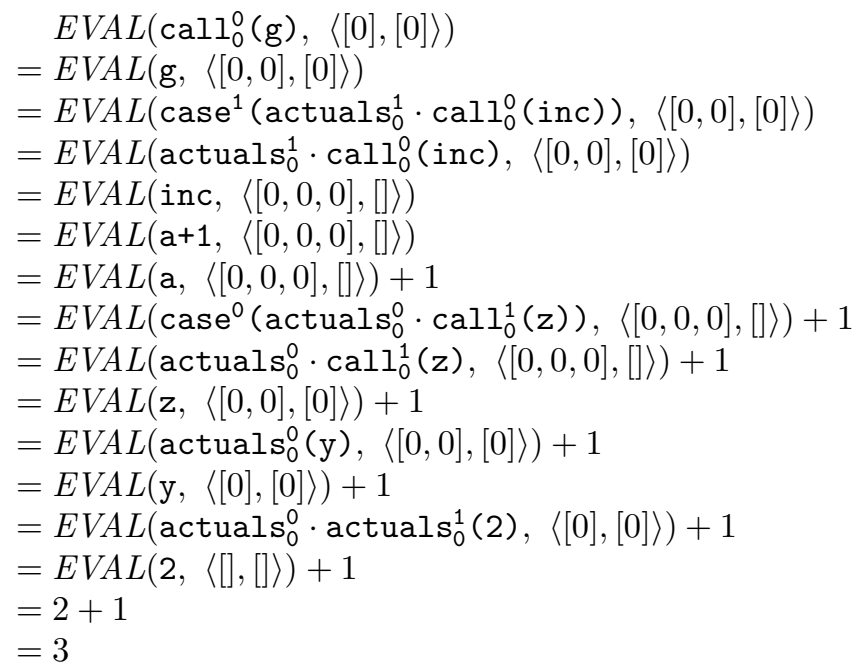

The second expression can be evaluated as follows: 


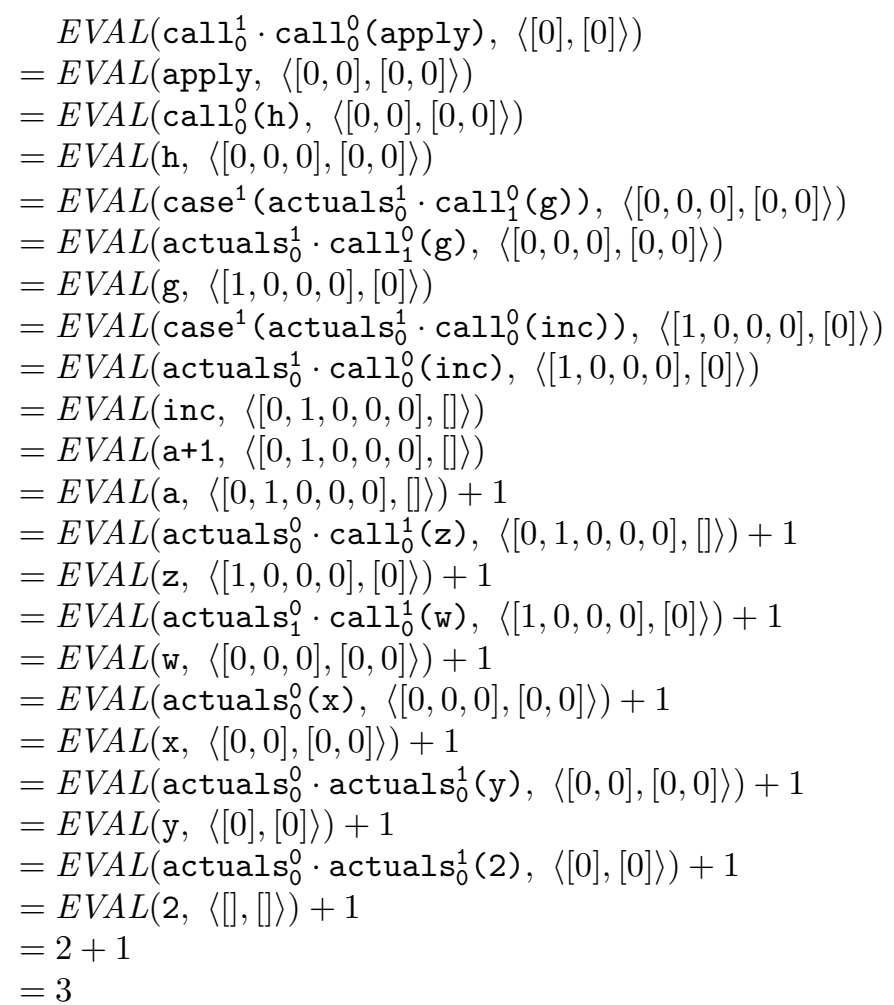

The final value of result is therefore $3 * 3=9$.

\section{The New Execution Model}

The execution model of section 2.2, based on the denotational semantics of the zero-order intensional language [RW99], is quite simple to understand and implement. However, the efficiency of a naïve implementation is doomed to be poor. Values that have been computed are often demanded again; an efficient implementation must memorize such values and not recompute them.

For example, consider the execution of the program in section 2.2. It can be argued that the evaluation process takes several unecessary steps. Some obvious recomputations occur, e.g. the variable y is demanded twice under the tag $\langle[0],[0]\rangle$ and computed twice, evidently yielding the same value. A more subtle case is the variable $\mathrm{g}$, a second-order formal parameter in the source program, which is demanded under the tags $\langle[0,0],[0]\rangle$ and $\langle[1,0,0,0],[0]\rangle$. Its evaluation inspects the second list in the tag, which is the same in both cases, and after some steps leads to the evaluation of inc. One may argue that an efficient implementation should memorize the fact that the evaluation of $g$ under a tag that has $[0]$ as a second list leads to the evaluation of inc and therefore avoid the intermediate steps. Both recomputation patterns tend to become very frequent in larger and more complex programs. 
In this section, we propose an evaluation model that can handle these issues. In this model, tags hold intermediate information (e.g. already computed values) which can be used to avoid recomputations. We first define a variation of the zero-order intensional language. Although modifications are required in the transformation proposed in [RW99] to produce final programs in this new target language, these modifications are minor. Subsequently, we define a denotational semantics for the new zero-order intensional language, which amounts to a new evaluation model.

\subsection{The modified zero-order intensional language}

The zero-order intensional language that we use as the target of the transformation is a variant of the NVIL language used in [RW99], which is the one that we used in section 2. The new syntax, however, stores additional information that will be used to improve the performance of the execution model. A program in the modified zero-order intensional language can be translated to NVIL in a straightforward way. However, the inverse cannot be achieved without additional information that can only be obtained during the transformation.

Definition 1 (Syntax). The syntax of the zero-order intensional language is defined recursively as follows, where $x$ is a variable identifier, $c$ is a constant and $m, n \in \mathbb{N}$.

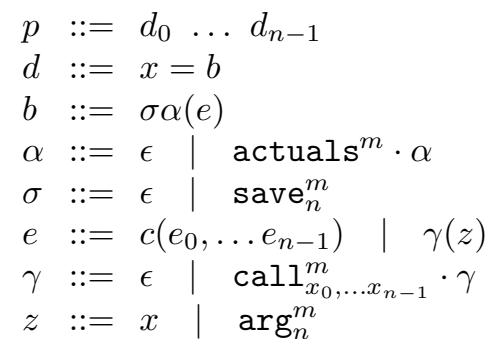

program

definition

body of definition

sequence of "actuals" operators

memorization operator

expression

sequence of "call" operators

variable

Notice that a variable $z$ can be either a variable identifier or a function argument, which is denoted by $\arg _{n}^{m}$. Variable identifiers are used for top-level variables, i.e. variables that have definitions in the source program. An argument of the form $\arg _{n}^{m}$ stands for the current function's $n$-th formal parameter of order $m$ as it was in the source higher-order program. The body of an intensional definition contains a memorization operator, which is either empty or of the form save ${ }_{n}^{m}$. This operator specifies whether the evaluation of the body must be followed by memorization of the result and where to; its purpose will become clear later. As an abuse of notation in favour of simplicity, the parentheses will be omitted after an empty sequence of call or actuals operators. Also, special constants (e.g. integers, arithmetic operators, etc.) will be written as is ordinary in programming languages.

The most striking differences between the modified zero-order intensional language and NVIL are the $\arg _{n}^{m}$ arguments, the lack of case construct, the lack of subscript for actuals operators and the presence of a finite sequence 
of variable identifiers instead of an integer counter as the subscript for call operators. To illustrate how all these work, let us consider again the simple second-order program that we used as an example in section 2.1.

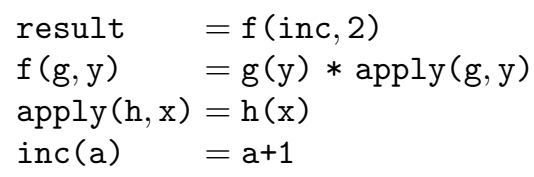

For purposes of comparison, we copy here the equivalent NVIL program from section 2.1, as produced by the intensional transformation [RW99].

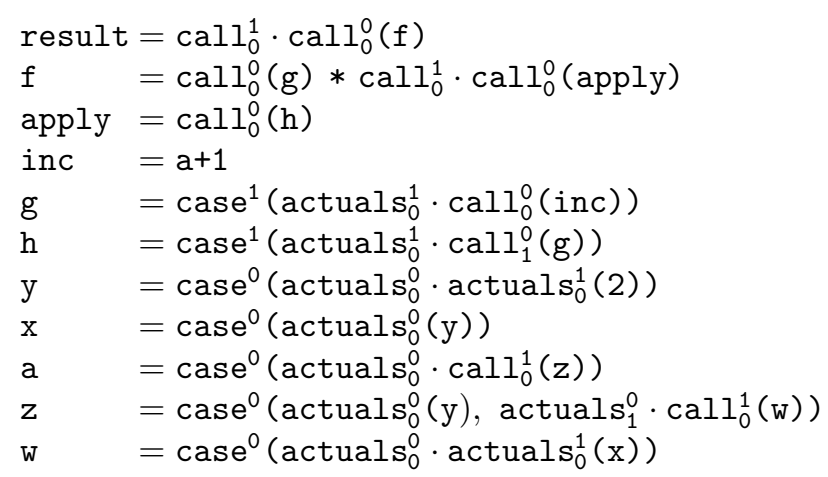

The modified transformation produces the following program in the modified zero-order intensional language.

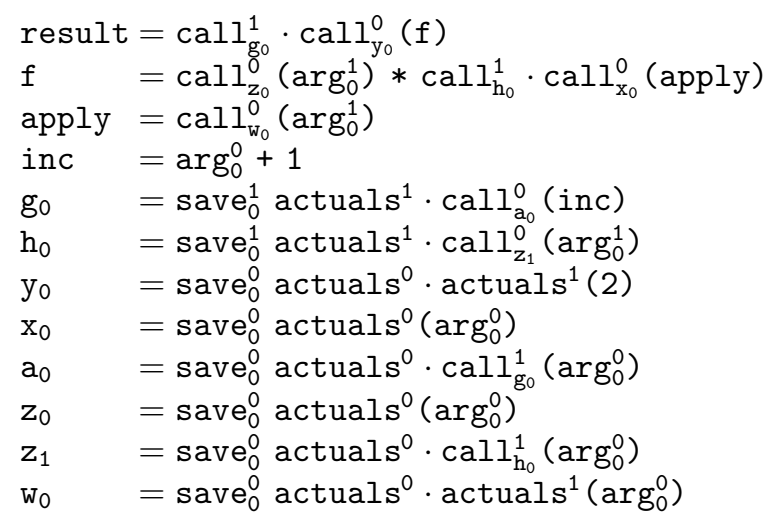

By comparing the two programs, we first notice that variable $g$ has been substituted with $\arg _{0}^{1}$. This happens because $g$ was $f$ 's first formal parameter of order 1 (remember that subscripted indices start with 0 ). Similarly, $\mathrm{x}$ has been substituted with $\arg _{0}^{0}$ as it was apply's first formal parameter of order 0 . We also notice that the case definitions have been removed. Each alternative has been introduced as a new definition, e.g. $z_{0}$ and $z_{1}$ instead of a single $z$. At the calling sites, the subscript of the call operator now contains a list of the formal parameters that were removed, each tagged with the original subscript, e.g. 
$\mathrm{call}_{\mathrm{g}_{0}}^{1}$ when the formal parameter $\mathrm{g}$ (of order 1 ) was removed from the first call to $f$. All elements of a list that is a subscript of call must appear in the left-hand side of newly introduced definitions. The subscripts of the actuals operators have been dropped and memorization operators have been added to all newly introduced definitions. For example, in the definition for $\mathrm{h}_{0}$ the memorization operator is save ${ }_{0}^{1}$ because $\mathrm{h}$ was apply's first formal parameter of order 1 .

\subsection{Semantics of execution}

The modified zero-order intensional language can be given a denotational semantics in a similar way as in [RW99]. In this paper we will define an $E V A L$ function (or, more precisely, a family of $E V A L$ functions) similar to the one described in section 2.2 .

We first introduce a new notion of tags which support memorization. A tag contains essentially all information that is available during execution, including memorized computation results. This information changes during the evaluation process. It is possible that the evaluation of expression $e_{1}$ affects the way in which another expression $e_{2}$ is evaluated, even if the two are unrelated, because of memorization. Therefore, tags must be treated as states in the new semantics, not as environments as in [RW99]. If Expr is the syntactic domain of expressions, $\mathbf{W}$ is the semantic domain of tags and Val the semantic domain of zero-order values (integer numbers, booleans, etc.), the signature of $E V A L$ must be:

\section{$E V A L: \mathbf{E x p r} \times \mathbf{W} \rightarrow \mathbf{V a l} \times \mathbf{W}$}

Similar functions are needed for the syntactic domains of variables and bodies of definitions. As an abuse of notation, we use the name $E V A L$ for them too.

A tag is an $M$-sequence of stacks. Each stack contains records, which are finite sequences of arguments. An argument can be either of the form value $(v)$, where $v$ is an element of $\mathbf{V a l}$, or of the form name $(x)$, where $x$ is a variable identifier. Intuitively, a record contains a sequence of the actual parameters that correspond to a function call; all parameters of order 0 are put together in a record that is placed in the first stack of the tag, and so on for parameters of higher order. When a record is first built, all its arguments are of the form name $(x)$. Their values are not yet known. When a zero-order argument is later computed, yielding the value $v$, it is replaced by value $(v)$ so as not to be recomputed in the future. On the other hand, when a higher-order argument is later computed, it may be replaced by another argument of the form name $(x)$ to avoid redundant evaluation steps.

A revised definition for $E V A L$ must redefine the semantics of intensional operators. Before going so far, we start with the easy cases. Assuming that there is a definition $x=b$ in the program, evaluating a variable identifier leads to evaluating the body of the definition.

$$
\operatorname{EVAL}(x, t)=E V A L(b, t)
$$

Also, assuming that a semantic function $\llbracket c \rrbracket$ exists for each constant $c$ of arity $n$, the semantics of constants is straightforward. Evaluation of a constant's operands is performed in left-to-right order. 


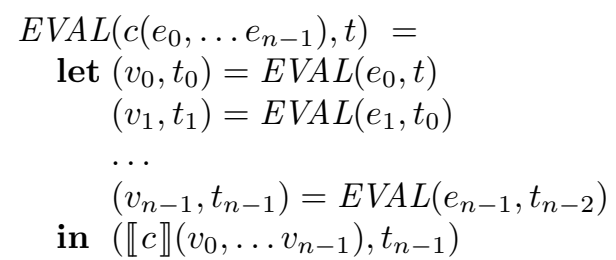

Function arguments are the next easier. To evaluate $\arg _{n}^{m}$, we take the record that is on top of the $m$-th stack of the tag. Assuming that this is a sequence of $K \geq n$ arguments (the transformation guarantees that), we inspect the $n$-th argument in that sequence. If it is a value, it is immediately reused. Otherwise, if it is a variable identifier, we evaluate it as previously.

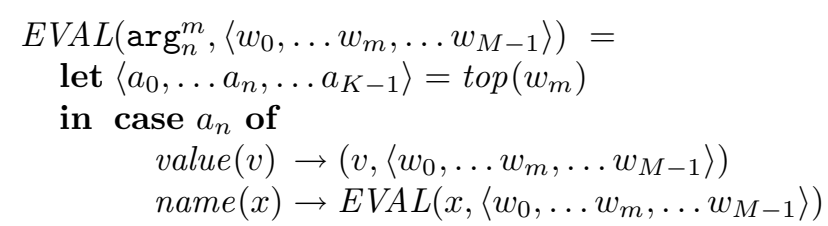

In the semantics of a $\mathrm{call}^{m}$ operator, we create a new record which contains the arguments that appear as its subscript. We put this record on top of the $m$-th stack of the tag and then evaluate the operand of the $\operatorname{call}^{m}$ operator under the new tag. When its evaluation is complete, we remove the top record from the $m$-th stack of the tag.

$$
\begin{aligned}
& E V A L\left(\operatorname{call}_{x_{0}, \ldots x_{n-1}}^{m}(e),\left\langle w_{0}, \ldots w_{m}, \ldots w_{M-1}\right\rangle\right)= \\
& \quad \text { let } r=\left\langle\operatorname{name}\left(x_{0}\right), \ldots \operatorname{name}\left(x_{n-1}\right)\right\rangle \\
& \quad\left(v,\left\langle w_{0}^{\prime}, \ldots w_{m}^{\prime}, \ldots w_{M-1}^{\prime}\right\rangle\right)=\operatorname{EVAL}\left(e,\left\langle w_{0}, \ldots \operatorname{push}\left(r, w_{m}\right), \ldots w_{M-1}\right\rangle\right) \\
& \quad \text { in } \quad\left(v,\left\langle w_{0}^{\prime}, \ldots \operatorname{pop}\left(w_{m}^{\prime}\right), \ldots w_{M-1}^{\prime}\right\rangle\right)
\end{aligned}
$$

The semantics of actuals $s^{m}$ operators that appear in the body of definitions is, in some sense, the inverse of that for $\mathrm{call}^{m}$ operators. We remove the top record from the $m$-th stack of the tag, but we store it so as to be able to put it back later. We evaluate the operand of the actuals operator (the remaining of a body of definition, possibly containing more actuals) under the new tag. When its evaluation is complete, we put back the stored record on top of the $m$-th stack of the tag.

$$
\begin{aligned}
& E V A L\left(\operatorname{actuals}{ }^{m}(b),\left\langle w_{0}, \ldots w_{m}, \ldots w_{M-1}\right\rangle\right)= \\
& \quad \text { let } r=\operatorname{top}\left(w_{m}\right) \\
& \quad\left(v,\left\langle w_{0}^{\prime}, \ldots w_{m}^{\prime}, \ldots w_{M-1}^{\prime}\right\rangle\right)=\operatorname{EVAL}\left(b,\left\langle w_{0}, \ldots \operatorname{pop}\left(w_{m}\right), \ldots w_{M-1}\right\rangle\right) \\
& \quad \text { in } \quad\left(v,\left\langle w_{0}^{\prime}, \ldots \operatorname{push}\left(r, w_{m}^{\prime}\right), \ldots w_{M-1}^{\prime}\right\rangle\right)
\end{aligned}
$$

The most complicated part of the semantics is memorization. First, notice that there is always an actual $\mathbf{s}^{m}$ operator following a save ${ }_{n}^{m}$ memorization operator. The evaluation of a body of the form save ${ }_{n}^{m}\left(\operatorname{actuals}^{m}(b)\right)$ is similar to that of actuals $s^{m}(b)$. The difference is that the record that is put back at the end on top of the $m$-th stack of the tag is not (necessarily) the same as the one that was removed in the beginning; the two may differ in the position $\arg _{n}^{m}$, which 
is updated as a result of evaluating the body. If $m=0$, i.e. the argument to be updated is zero-order, then the value that was computed replaces the previous contents of $\arg _{n}^{m}$. Otherwise, if the argument is higher-order, the updated argument depends on the syntactic form of $b$. If $b$ ends with a variable identifier, then this replaces the previous contents of $\arg _{n}^{m}$. On the other hand, if it ends with a (possibly different) function argument, this argument in the tag that resulted from the evaluation of $e$ replaces the previous contents of $\arg _{n}^{m}$.

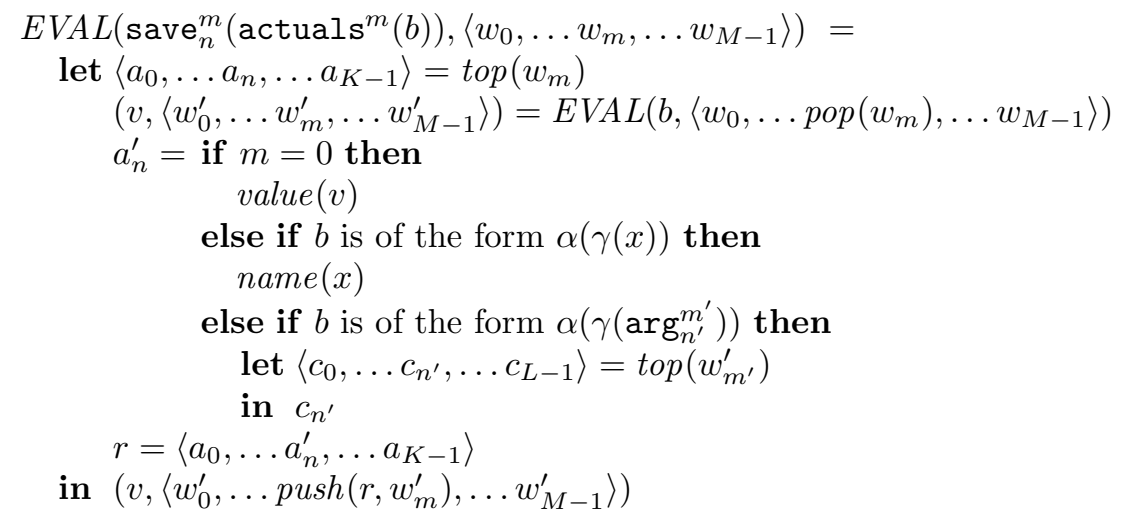

\section{Implementation on Stock Hardware}

The success of an execution model depends primarily on how efficiently it can be implemented. In this section we outline a quite efficient implementation of the model that was presented in section 3 on stock hardware. Our implementation presents similarities to the traditional use of activation records, which are used to hold a function's actual parameters and are organized as a stack in the computer's memory. In our approach, which is an extension of the scheme proposed in [RW94b], there are two main differences: (i) activation records are lazy, i.e. the actual parameters are filled in upon demand; and (ii) the construction and destruction of activation records is controlled by the presence of intensional operators in the zero-order program.

The most crucial and at the same time tricky issue in the implementation of the execution model is the representation of tags, records and arguments and the implementation of intensional operators. We represent stacks of records by linked lists, i.e. we add to each record a pointer to the record that is immediately below on the stack. We represent a tag by a set of $M$ pointers $\left\{t_{0}, \ldots t_{M-1}\right\}$ that point to the first elements of the lists representing the stacks. For the representation of a record's arguments, we use one bit to distinguish between a $\operatorname{value}(v)$ and a name $(x)$; the representation of $v$ or $x$ follows. A variable identifier $x$ can be represented by a pointer to the code that implements the corresponding definition.

There is an obvious similarity between the semantics of call and actuals operators and traditional stack-based activation records: a call can be viewed as putting a record on the stack, evaluating the "function body" and then removing 


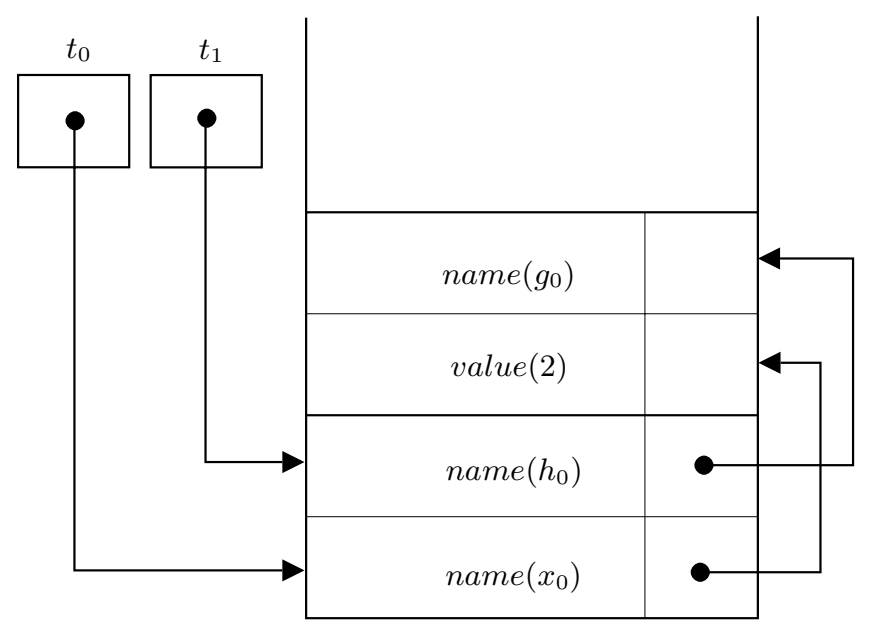

Fig. 1. An example of lazy activation records.

the record from the stack. On the other hand, actuals ignores the record that is on top of the stack and switches the context to the one immediately below, in order to evaluate its body; then it switches the context back to what it was. This remark allows us to group the records of successive calls, in the sense of the execution model of section 3, in lazy activation records that we place on a stack in the computer's memory. The form of some lazy activation records that appear during execution of the example in section 2.2 is shown in Fig. 1.

As execution begins, the first lazy activation record that is constructed is the one for $f$, with $g$ and $y$ as arguments (the top one in the figure). After some time, $\mathrm{y}$ is evaluated giving the value 2 , which replaces the argument on the stack. When apply is called, with arguments $\mathrm{h}$ and $\mathrm{x}$, a new lazy activation record is placed on the stack (the stack grows downward in the figure). Notice at this point of execution the pointers $\left\langle t_{0}, t_{1}\right\rangle$ in the current tag, pointing to two separate linked lists, one for each dimension.

\section{Performance Comparison and Related Work}

In this section we briefly summarize other implementation techniques for higherorder functional programming languages and compare our implementation with some other known implementations in terms of performance.

The traditional implementation technique for lazy functional languages is based on graph reduction [SPJ87]. Great effort has been made over the years for efficient implementations [Joh84] and optimizations. We consider the well-known compilers for Haskell (ghc and hbc) and Clean as representatives of the several existing implementations of graph reduction. They are mature compilers and implement more features than we discuss in this paper. Despite the restrictions 
of our technique, reduction-based compilers provide a good reference point for evaluating the efficiency and viability of the intensional approach.

We are also interested in comparing the new execution model with a hashingbased implementation of the intensional approach [RW93]. The same idea is used for implementing intensional languages, e.g. Lucid [Wad91]. We use as a reference point a more recent implementation of the intensional approach that uses a garbage-collecting warehouse for memorizing intermediate values [Gri04].

The experimental compiler that we developed ${ }^{3}$ is based on the execution model and implementation technique described in sections 3 and 4 [Cha05]. It compiles source programs, written in a higher-order functional language, to lowlevel $\mathrm{C}$ code. At its present state, our compiler does not try to optimize function bodies (a number of such optimizations are performed by the $\mathrm{C}$ compiler) and does not eliminate tail-recursive calls, which occur very often in the final $\mathrm{C}$ program.

We used the following programs as benchmarks for comparing the performance of the aforementioned implementations. The order of each program is shown in parentheses.

nofib (1): A standard benchmark, computing a variation of Fibonacci numbers in exponential time, for $n=30$.

queens (1): A program solving the $N$ queens' problem for $N=9$, using large integer numbers to encode chessboard configurations.

primes (1): A program computing prime numbers up to 7500 .

ack (1): A program computing Ackermann's function for $m=3$ and $n=9$.

tak (2): A program computing a second-order variation of Takeuchi's function for $x=24, y=16$ and $z=8$.

ntak (3): A program computing a third-order variation of Takeuchi's function for $x=24, y=16$ and $z=8$.

integrate (3): A program that computes several integrals using various methods of integration.

church (4): A program that performs some integer arithmetic with Church numerals.

Table 1 shows the execution times obtained by executing the benchmark programs. For a fair comparison, optimizations such as strictness analysis were disabled in compilers that perform them. Moreover, when there was an option to produce $\mathrm{C}$ instead of native code, it was preferred.

First of all, it is clear that our implementation is a significant improvement over the hashing-based implementation, as it outperforms it by several orders of magnitude in most cases. For most benchmarks, our implementation is as fast as the reduction-based systems. In some cases, usually in first-order programs, our implementation appears to outperform Haskell's compilers. Moreover, the behaviour of our technique in programs with a high number of function calls

\footnotetext{
${ }^{3}$ Our compiler and the sources of the benchmark programs can be obtained from:

ftp://ftp.softlab.ntua.gr/pub/users/nickie/software/lar.tar.gz
} 
Table 1. Execution times for benchmark programs.

\begin{tabular}{|c|c|c|c|c|c|}
\hline & \multirow{2}{*}{\multicolumn{2}{|c|}{$\begin{array}{c}\text { Intensional } \\
\text { Stack-based Hashing-based }\end{array}$}} & \multicolumn{3}{|c|}{ Reduction-based } \\
\hline & & & $\mathrm{GHC}$ & $\mathrm{HBC}$ & Clean \\
\hline \multicolumn{6}{|c|}{ First order } \\
\hline nofib & 0.080 & 2.650 & 0.550 & 1.363 & 0.005 \\
\hline queens & 0.050 & 2.170 & 0.320 & 0.837 & 0.005 \\
\hline primes & 0.030 & 0.820 & 0.435 & 1.232 & 0.010 \\
\hline ack & 1.270 & 40.580 & 2.453 & 6.889 & 0.270 \\
\hline \multicolumn{6}{|c|}{ Higher order } \\
\hline tak (2) & 0.310 & 43.610 & 0.372 & 0.664 & 0.090 \\
\hline ntak (3) & 0.750 & 126.410 & 1.452 & 4.854 & 0.240 \\
\hline integrate (3) & 0.300 & too long! & 0.765 & 1.373 & 0.050 \\
\hline church (4) & 0.010 & 0.040 & 0.001 & 0.001 & 0.001 \\
\hline
\end{tabular}

(e.g. nofib, ack, tak, ntak) is competitive; it can be significantly improved by implementing tail recursion elimination. On the other hand, in most cases, Clean's compiler produces faster code than all the others. A possible explanation is that it produces native code instead of $\mathrm{C}$ and makes some tail-call transformation to obtain more efficient code. Garbage collection and the handling of heap-allocated closures is one of the reasons that add a time overhead to reduction-based implementations. Our technique operates using a stack; that is, no garbage collecting is needed as long as no heap is used. Garbage collection may become necessary when complex data structures are supported in the source language.

In general, the performance of our implementation is competitive to that of current efficient reduction-based implementations. Furthermore, no performance degradation occurs when the order of program increases, in contrast to [RW94b].

\section{Conclusion and Future Work}

The intensional transformation has long been proposed as an alternative implementation technique for lazy functional languages. Its main idea is to transform a higher-order source functional program into an equivalent zero-order intensional program. The purpose of this paper was to assess the efficiency and viability of this technique. We have proposed an efficient model for executing the transformed intensional zero-order programs and compared a prototype implementation of this model with other techniques, reduction-based or intensional. The results indicate that the intensional technique can be as efficient as most modern implementation techniques for lazy functional languages, for the class of programs that can be transformed.

Although our results are very promising, this technique is still far from being able to implement a fully featured lazy functional language. Apart from the 
known shortcomings of the intensional transformation, imposing restrictions on the source programs [RW99], a long list of features remain to be supported by this technique. One of the most important missing features is arbitary data structures. Furthermore, optimizations of our implementation such as strictness analysis and tail recursion elimination must be investigated as future work.

\section{References}

[Cha05] A. Charalambidis. An Efficient Technique for Implementing Lazy Functional Programming Languages. Diploma Dissertation. Department of Informatics and Telecommunications, University of Athens, June 2005.

[Gri04] A. Grivas. Implementation of Functional Languages using the Branching Dimensions Transformation. Diploma Dissertation. School of Electrical and Computer Engineering, National Technical University of Athens, October 2004.

[Joh84] T. Johnsson. Efficient Compilation of Lazy Evaluation. in Proceedings of ACM Compiler Construction, Montreal, Canada, June 1984, ACM SIGPLAN Notices, 19(6), pp. 58-69.

[SPJ87] S. L. Peyton Jones. The Implementation of Functional Programming Languages. Prentice Hall, 1987.

[Ron94] P. Rondogiannis. Higher-Order Functional Languages and Intensional Logic. PhD thesis, Department of Computer Science, University of Victoria, Canada, 1994.

[RW93] P. Rondogiannis and W. W. Wadge. A Dataflow Implementation Technique for Lazy Typed Functional Languages. In Proceedings of the Sixth International Symposium on Lucid and Intensional Programming, pages 23-42, 1993.

[RW94a] P. Rondogiannis and W. W. Wadge. Compiling Higher-Order Functions for Tagged-Dataflow. In Proceedings of the IFIP International Conference on Parallel Architectures and Compilation Techniques, pages 269-278. NorthHolland, August 1994.

[RW94b] P. Rondogiannis and W. W. Wadge. Higher-Order Dataflow and its Implementation on Stock Hardware. In Proceedings of the ACM Symposium on Applied Computing, pages 431-435. ACM Press, 1994.

[RW97] P. Rondogiannis and W. W. Wadge. First-Order Functional Languages and Intensional Logic. Journal of Functional Programming, 7(1):73-101, January 1997.

[RW99] P. Rondogiannis and W. W. Wadge. Higher-Order Functional Languages and Intensional Logic. Journal of Functional Programming, 9(5):527-564, September 1999.

[Wad91] W. W. Wadge. Higher-Order Lucid. In Proceedings of the Fourth International Symposium on Lucid and Intensional Programming, 1991.

[Yag84] A. A. Yaghi. The Intensional Implementation Technique for Functional Languages. PhD thesis, Department of Computer Science, University of Warwick, Coventry, UK, 1984. 